Article

\title{
Spectral and Polarization Signatures of Relativistic Shocks in Blazars
}

\author{
Markus Böttcher 1,2 \\ 1 Centre for Space Research, North-West University, Potchefstroom 2520, South Africa; \\ Markus.Bottcher@nwu.ac.za \\ 2 Department of Physics and Astronomy, Ohio University, Athens, OH 45701, USA
}

Academic Editors: Jose L. Gómez, Alan P. Marscher and Svetlana G. Jorstad

Received: 7 July 2016; Accepted: 30 August 2016; Published: 2 September 2016

\begin{abstract}
Relativistic shocks are one of the most plausible sites of the emission of strongly variable, polarized multi-wavelength emission from relativistic jet sources such as blazars, via the diffusive shock acceleration (DSA) of relativistic particles. This paper summarizes recent results on a self-consistent coupling of diffusive shock acceleration and radiation transfer in blazar jets. We demonstrate that the observed spectral energy distributions (SEDs) of blazars strongly constrain the nature of hydromagnetic turbulence responsible for pitch-angle scattering by requiring a strongly energy-dependent pitch-angle mean free path. The prominent soft X-ray excess ("Big Blue Bump") in the SED of the BL Lac object AO 0235+164 can be modelled as the signature of bulk Compton scattering of external radiation fields by the thermal electron population, which places additional constraints on the level of hydromagnetic turbulence. It has further been demonstrated that internal shocks propagating in a jet pervaded by a helical magnetic field naturally produce polarization-angle swings by $180^{\circ}$, in tandem with multi-wavelength flaring activity, without requiring any helical motion paths or other asymmetric jet structures. The specific application of this model to 3C279 presents the first consistent simultaneous modeling of snap-shot SEDs, multi-wavelength light curves, and time-dependent polarization signatures of a blazar during a polarization-angle (PA) rotation. This model has recently been generalized to a lepto-hadronic model, in which the high-energy emission is dominated by proton synchrotron radiation. It is shown that in this case, the high-energy (X-ray and $\gamma$-ray) polarization signatures are expected to be significantly more stable (not showing PA rotations) than the low-energy (electron-synchrotron) signatures.
\end{abstract}

Keywords: active galaxies: BL Lac objects; jets and bursts; radiation mechsnisms: polarization

\section{Introduction}

Ever since the pioneering work of Marscher \& Gear [1], relativistic shocks have been considered one of the leading contenders for the location of relativistic particle acceleration, resulting in the observed rapidly variable, often highly polarized multi-wavelength emission from blazars. Models focusing on the multi-zone radiative transfer problem(given efficient relativistic shock acceleration) have reached an increasing level of sophistication (e.g., [2-10]), resulting in successful fits to multi-wavelength spectral energy distributions (SEDs) and light curves of individual flares in blazars. In all cited works, however, the details of particle acceleration are not specifically addressed, but shock acceleration is assumed to result in the injection of relativistic particles, typically with a power-law distribution in energy. In a recent work, Baring et al. [11] have coupled the Monte-Carlo simulations of diffusive shock acceleration of Summerlin and Baring [12] with radiative transfer routines of Böttcher et al. [13]. Their results are briefly summarized in Section 2, focusing on implications for the nature of hydromagnetic turbulence facilitating pitch-angle scattering. 
An important additional observable that is usually neglected in detailed radiative transfer simulations for blazars is the linear polarization of synchrotron (and possibly also Compton) emission. A peculiar feature that has been observed in several cases (e.g., [14,15]) consists of large polarization-angle (PA) swings by $\geq 180^{\circ}$ associated with multi-wavelength flaring activity. With very few exceptions (e.g., [16,17]), theoretical models for synchrotron polarization variations are purely geometrical, often with an ad hoc assumption of helical pattern motions guided by helical magnetic fields, without consistent considerations of radiation physics or particle dynamics (e.g., [18]). In this context, it should be pointed out that most works modeling the SEDs of blazars in detail (e.g., $[13,19])$ indicate that in the high-energy emission region, blazar jets are particle dominated (i.e., with sub-equipartition magnetic fields), in which case the relativistic plasma is not expected to be guided by the magnetic fields, but instead the magnetic fields will follow the plasma motion. Note furthermore that in the case of polarization and flux variations dominated by viewing-angle changes, the variability is expected to be dominated by Doppler-factor changes. In this case, the variability is quasi-achromatic, with peak flux variations of individual radiation components, $\delta\left(v F_{v}^{p}\right)$ related to peak frequency variations $\delta v_{p}$ as $\delta\left(v F_{v}^{p}\right) \propto\left(\delta v_{p}\right)^{4}$. This contradicts the strongly frequency-dependent flux variability patterns normally observed in blazars.

One of the first models that consistently treats radiation physics with time-dependent synchrotron polarization signatures is the Turbulent Extreme Multi-Zone Model (TEMZ) [16]. Here, polarization variations are the result of the energization of a small number of radiative cells, each with randomly oriented (turbulent) magnetic fields. This model has proven very successful in reproducing stochastic variations of polarization characteristics, and may occasionally even lead to organized PA swings. In this model, PA swings are not expected to be systematically associated with multi-wavelength flaring activity, in agreement with the statistical analysis of a large number of PA rotations observed by RoboPol [20]. However, there is also a significant number of events in which PA rotations do occur in clear correlation with multi-wavelength flares (e.g., [14,15]). For this latter case, Zhang et al. [17,21] have developed a model self-consistently tracing particle dynamics, radiation transfer, and resulting polarization characteristics in a shock-in-jet model with an ordered, helical magnetic field. Their results will be briefly summarized in Section 3. This model has recently been generalized to a lepto-hadronic model, which will be presented in Section 4 .

\section{Diffusive Shock Acceleration in Relativistic Jets}

Diffusive shock acceleration (DSA) is generally considered to be one of the most efficient modes of particle acceleration at relativistic shocks. Detailed Monte-Carlo (MC) simulations of DSA at relativistic oblique shocks have recently been carried out by Summerlin and Baring [12]. In the DSA scenario, the Fermi-I acceleration process is facilitated by pitch-angle scattering, causing the guiding centers of particles spiraling along magnetic field lines to reverse their direction of motion in a stochastic manner. Pitch-angle scattering is parameterized through the pitch-angle mean-free path $\lambda_{\mathrm{mfp}}$ as a (generally energy-dependent) multiple $\eta(p)$ of the particle's gyro radius, $r_{g}=p c /(q B)$, where $p$ is the particle's momentum, such that $\lambda_{\operatorname{mfp}}=\eta(p) r_{g}$. The energy dependence of the mean-free-path parameter $\eta$ is parameterized as a power-law in the particle's momentum, $\eta(p)=\eta_{1} p^{\alpha-1}$, so that $\lambda_{\mathrm{mfp}} \propto p^{\alpha}$ and $\eta_{1}$ describes the mean free path in the non-relativistic limit, $\gamma \rightarrow 1$.

In a recent paper, Baring et al. [11] have now coupled the MC simulations of DSA to radiative transport, adopting the routines of [13]. To obtain realistic thermal + non-thermal particle distributions, a high-energy cut-off $\left(\gamma_{\max }\right)$ of the non-thermal particle spectra has been evaluated by balancing the acceleration time scale $t_{\mathrm{acc}}\left(\gamma_{\max }\right)=\eta\left(\gamma_{\max }\right) t_{\mathrm{gyr}}\left(\gamma_{\max }\right)$ with the radiative energy loss time scale. In the presence of dominant synchrotron losses, this will lead to a synchrotron peak energy $E_{\mathrm{sy}} \sim 240 \delta \eta^{-1}\left(\gamma_{\max }\right) \mathrm{MeV}$, independent of $B$, where $\delta$ is the Doppler factor. Thus, in order to reproduce a synchrotron peak in the IR to soft X-rays (as typical for blazars), $\eta\left(\gamma_{\max }\right)$ has to assume values of $\sim 10^{4}-10^{8}$, depending on the location of the synchrotron peak. On the other hand, as demonstrated in [12], $\eta_{1}$ must be significantly smaller than this in order to obtain efficient injection 
of particles out of the thermal pool into the non-thermal acceleration process. This indicates that $\eta(\gamma)$ must be strongly energy dependent.

Figure 1 shows a fit to the SED of the high-frequency peaked BL Lac object (HBL) Mrk 501 (data from [22]), which exhibits its characteristic synchrotron peak at soft X-ray energies. This, along with efficient acceleration of particles out of the thermal pool, could be achieved with a choice of $\eta_{1}=100$ and $\alpha=1.5$; i.e., $\eta(\gamma)=100 \gamma^{0.5}$. Other parameters are typical of leptonic synchrotron self-Compton fits to HBLs in general, and Mrk 501 in particular. For details, see [11].

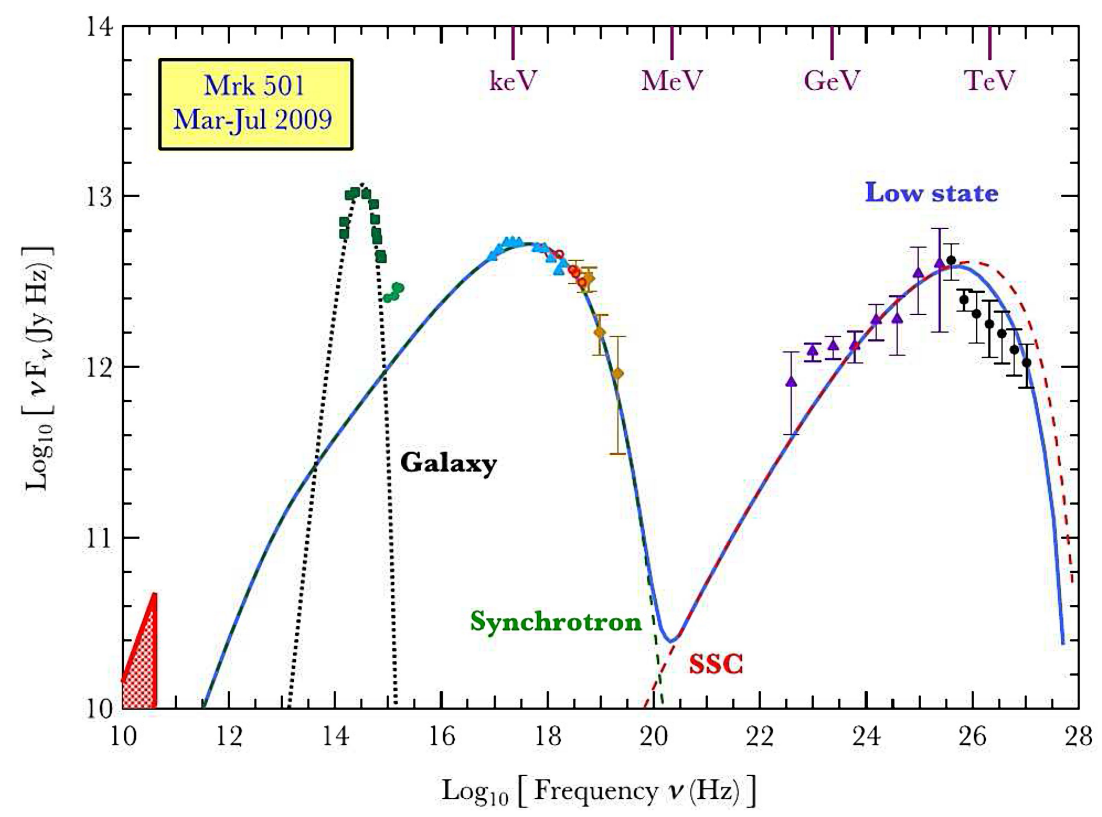

Figure 1. Fit to the spectral energy distribution (SED) of the high-frequency peaked BL Lac object (HBL) Mrk 501 (data from [22]), with $\eta_{1}=100$ and $\alpha=1.5$. From [11].

An example of a low-frequency-peaked BL Lac object (LBL) is shown in Figure 2 (with data from [23]). In this case, a much more extreme energy dependence of $\eta$ is required in order to reproduce the observed synchrotron peak in the IR: $\eta_{1}=225$ and $\alpha=3$; i.e., $\eta(\gamma)=225 \gamma^{2}$. In this case, the non-relativistic limit $\eta_{1}$ is very well constrained by the proposition that the soft $X$-ray excess indicated by the steep Swift-XRT 2-10 keV spectrum is produced by the bulk Compton mechanism [24], where the thermal population of electrons Compton up-scatters the external radiation field (dominated by Broad Line Region emission) by a factor $\sim \Gamma^{2}$, where $\Gamma$ is the bulk Lorentz factor of the post-shock emission region.

The implied strong energy dependence of the pitch-angle mean free path allows us to diagnose the characteristics of hydromagnetic turbulence in the blazar jet. Specifically, pitch-angle scattering is most efficient for particles interacting gyro-resonantly with plasma waves of the same (co-moving) frequency as their own gyro-frequency. This translates into gyro-resonant particle-wave interactions with waves having a wave number $k_{\|}$in the propagation direction along the guiding magnetic field, given by $k_{\|}=\omega_{B} /(\gamma v|\mu|)$, where $\omega_{B}$ is the particle's gyro-frequency, $v$ its velocity, and $\mu$ the cosine of its pitch angle. To first order, this implies that the resonant wavelength for a particle with energy $\gamma$ scales as $\lambda_{\text {res }} \propto \gamma$. Consequently, the highest-energy particles will interact primarily with the longest wavelength plasma waves (smallest $k_{\|}$). Thus, two effects are expected to combine to suppress such gyro-resonant interactions for very long wavelengths: (1) For wavelengths approaching (and exceeding) the "stirring scale" on which plasma turbulence is induced, the energy density contained in such waves is rapidly decreasing with increasing wavelengths (see Figure 3); (2) the highest energy particles will probe large volumes behind the shock front, where the level of turbulence is expected to decrease with 
distance from the shock. These effects provide a natural explanation of the strong energy dependence of the pitch-angle mean free path which we find in our analyses. The internal consistency of this picture provides support for DSA as the dominant particle acceleration mechanism in blazar jets.

AO $0235+164$

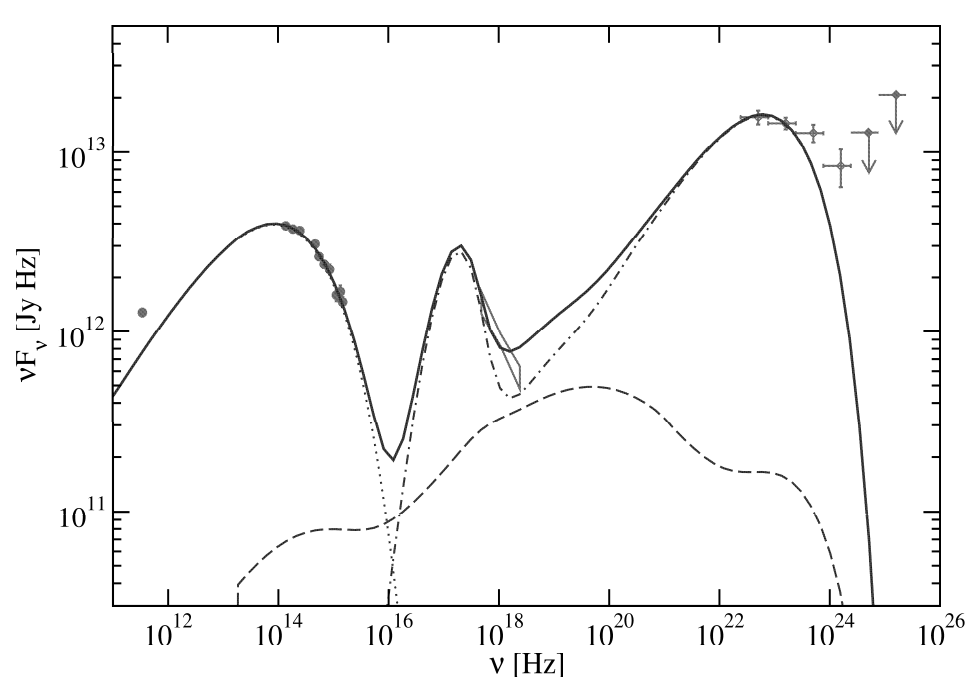

Figure 2. Fit to the SED of the low-frequency-peaked BL Lac object (LBL) AO 0235+164 (data from [23]), with $\eta_{1}=225$ and $\alpha=3$. Note that the soft X-ray excess (Big Blue Bump) is reproduced by the bulk Compton emission of the thermal electron population. From [11].

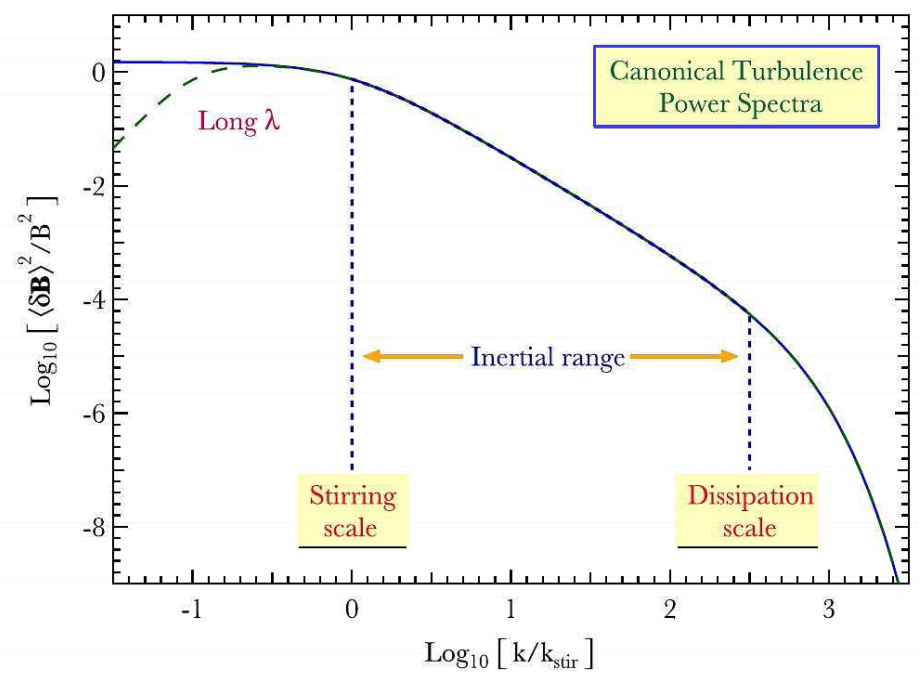

Figure 3. Sketch of a magneto-hydrodynamic turbulence spectrum plausibly expected in the post-shock region. Waves are excited at the longest wavelengths corresponding to the stirring scale, and cascade down to smaller scales, down to the dissipation scale. From [11].

\section{Polarization-Angle Swings}

As an alternative to the purely geometry-based models of helical pattern motions for the optical PA swings observed in several blazars (as discussed in Section 1) correlated with multi-wavelength flaring activity, Zhang et al. [17,21] have proposed a model that does not require any asymmetric jet features and/or pattern motions. It is the first model that consistently considers particle dynamics and time- and polarization-dependent radiation transport, along with all relevant light-travel time effects. 
Most importantly, Zhang et al. [17] have shown that careful consideration of all light travel time and radiation-transfer effects naturally leads to $\sim 180^{\circ}$ PA rotations when a shock propagates through an active region in a jet pervaded by a helical magnetic field.

A spectacular success of this model is provided by its application to the prominent PA rotation and multi-wavelength flare event of 3C279 in 2009 [14], as presented in [21]. Here, the authors have presented for the first time fits to snap-shot SEDs, multi-wavelength light curves, and time-dependent optical synchrotron polarization signatures (Polarization Degree and Polarization Angle), within one consistent model, properly treating particle dynamics and polarization-dependent radiation transfer. A key result of this study was that these fits required a decreasing magnetic field strength along with enhanced particle acceleration, indicative of magnetic energy dissipation as the driving mechanism for the observed flaring activity. For details of the simulations and the parameters required for the fit presented in Figure 4, see [21].
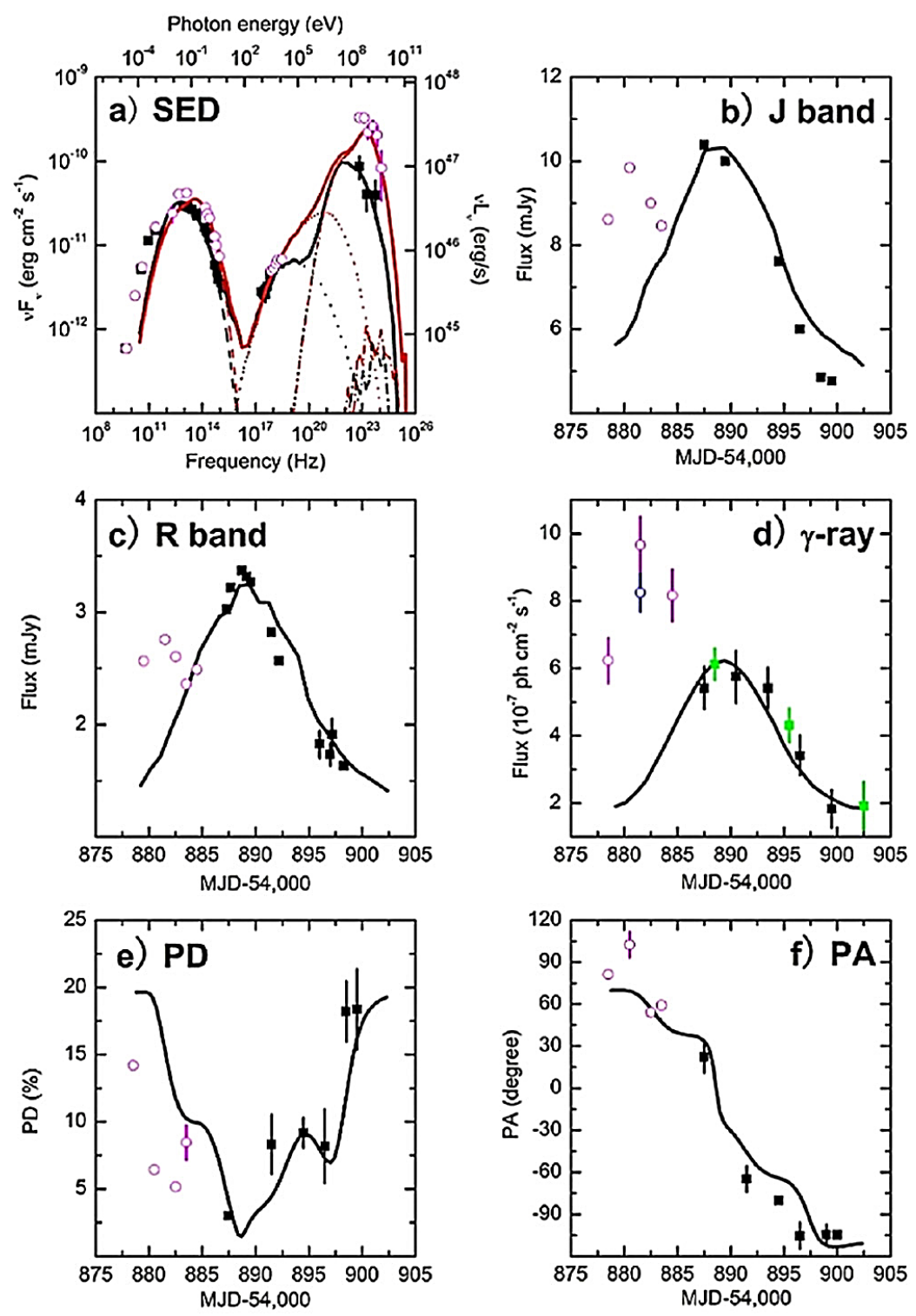

Figure 4. The multiwavelength flare + position angle (PA) swing event of 3C279 in 2009 [14]: Simultaneous fits to (a) snap-shot SEDs; (b-d) multi-wavelength light curves; and (e,f) time-dependent optical polarization characteristics. PD: polarization degree. From [21]. 


\section{A Polarization-Dependent Multi-Zone Lepto-Hadronic Internal Shock Model}

While the electron-synchrotron origin of the low-energy (radio-optical/UV/X-ray) SED component of blazars is well established, the nature of the high-energy (X-ray- $\gamma$-ray) emission is less clear. Both leptonic scenarios, where the $\gamma$-rays result from Compton scattering of various radiation fields by the same leptons that produce the low-energy synchrotron emission; and hadronic scenarios, where the high-energy emission is produced by ultrarelativistic protons (via proton synchrotron radiation and/or photo-pion production and subsequent pion decay), are viable and being actively investigated. However, due to the significantly more complex nature of hadronic interactions (and subsequent pion and muon decay and cascading), hadronic models are only now beginning to be investigated in a time-dependent manner, and to the author's knowledge, all published works on hadronic blazar models are single-zone in nature, most of them time-independent (e.g., [13,25-31]).

A fully time-dependent single-zone lepto-hadronic blazar model was recently developed by Diltz et al. [32], where the particle dynamics of all decay products is properly taken into account, including pion and muon synchrotron radiation. The proton evolution and proton synchrotron radiation routines of that model have recently been incorporated into the polarization-dependent radiation-transfer model described in the previous section. This provides us with the first 3D time- and polarization-dependent shock-in-jet model incorporating proton synchrotron radiation. As detailed cross-zone radiation transport is not included in the model, synchrotron self-Compton radiation and photo-pion induced processes are neglected, which restricts the model to a parameter regime in which the magnetic energy density greatly exceeds the co-moving photon energy density (for detailed parameter estimates resulting from this restriction, see [33]).

In [33], we presented a detailed parameter study of our proton-synchrotron shock-in-jet model. We found that the variability characteristics of the polarization signatures (Polarization Degree and Polarization Angle) depend critically on the relation between the light crossing time across the active region, $t_{\mathrm{cr}}$, and the radiative cooling time scale $t_{\mathrm{cool}}$ of the highest-energy protons. Figure 5 illustrates the results in the case $t_{\mathrm{cr}}<t_{\text {cool }}$ for a flaring scenario in which more efficient particle acceleration is accompanied by a reduction of the magnetic field (magnetic energy dissipation). The jet is pervaded by a helical magnetic field with a similar baseline configuration, as used in $[17,21]$ to reproduce PA swings correlated with multi-wavelength flaring activity in a leptonic model context.

The baseline parameter set leads to an SED that is characteristic of a low-frequency peaked blazar so that, in this model, the X-ray emission is dominated by the same proton-synchrotron radiation component responsible for the $\gamma$-rays. The figure illustrates that this scenario leads to significant multi-wavelength flaring activities, and that the high-energy (X-ray through $\gamma$-ray) emission is expected to be highly polarized, to a similar degree as the optical (electron-synchrotron) emission during the quiescent state.

Most importantly, the bottom-left panel of Figure 5 shows that the PA of the high-energy emission is expected to stay relatively stable throughout the shock-induced flare, while the optical emission exhibits the well-known $180^{\circ} \mathrm{PA}$ swing. The reason for this is the significantly longer radiative cooling time scale for protons compared to electrons, due to which a much larger region behind the shock remains active. This is also reflected in the significantly longer decay time scale of the X-ray through $\gamma$-ray emission compared to the electron-synchrotron (radio-UV) emission.

This has important consequences for the prospects of future X-ray or $\gamma$-ray polarimetry missions (e.g., XIPE, PolSTAR, PANGU) to detect high-energy polarization from blazars. During the required long integration time of these instruments to potentially detect X-ray (or $\gamma$-ray) polarization from blazars, the optical PA may very well vary by significant amounts and possibly exhibit large-angle PA swings. If the X-ray/ $\gamma$-ray PA were behaving in the same way, and this was not properly accounted for in the data analysis of high-energy polarimeters, this would completely smear out any polarization signatures. Instead, we here find that the PA of proton-synchrotron-dominated X-ray and $\gamma$-ray emission may quite plausibly remain stable, even if the optical PA exhibits large-angle swings, thus greatly enhancing the chances of a positive detection of these high-energy polarization signatures. 
As pointed out by Zhang and Böttcher [34], the possibly substantial high-energy polarization degrees predicted by hadronic models are in stark contrast to the very low degree of $X$-ray and $\gamma$-ray polarization expected in leptonic models. Thus, our results reinforce future prospects of using $X$-ray and $\gamma$-ray polarization as a diagnostic tool to distinguish leptonic from hadronic high-energy emission in blazars.
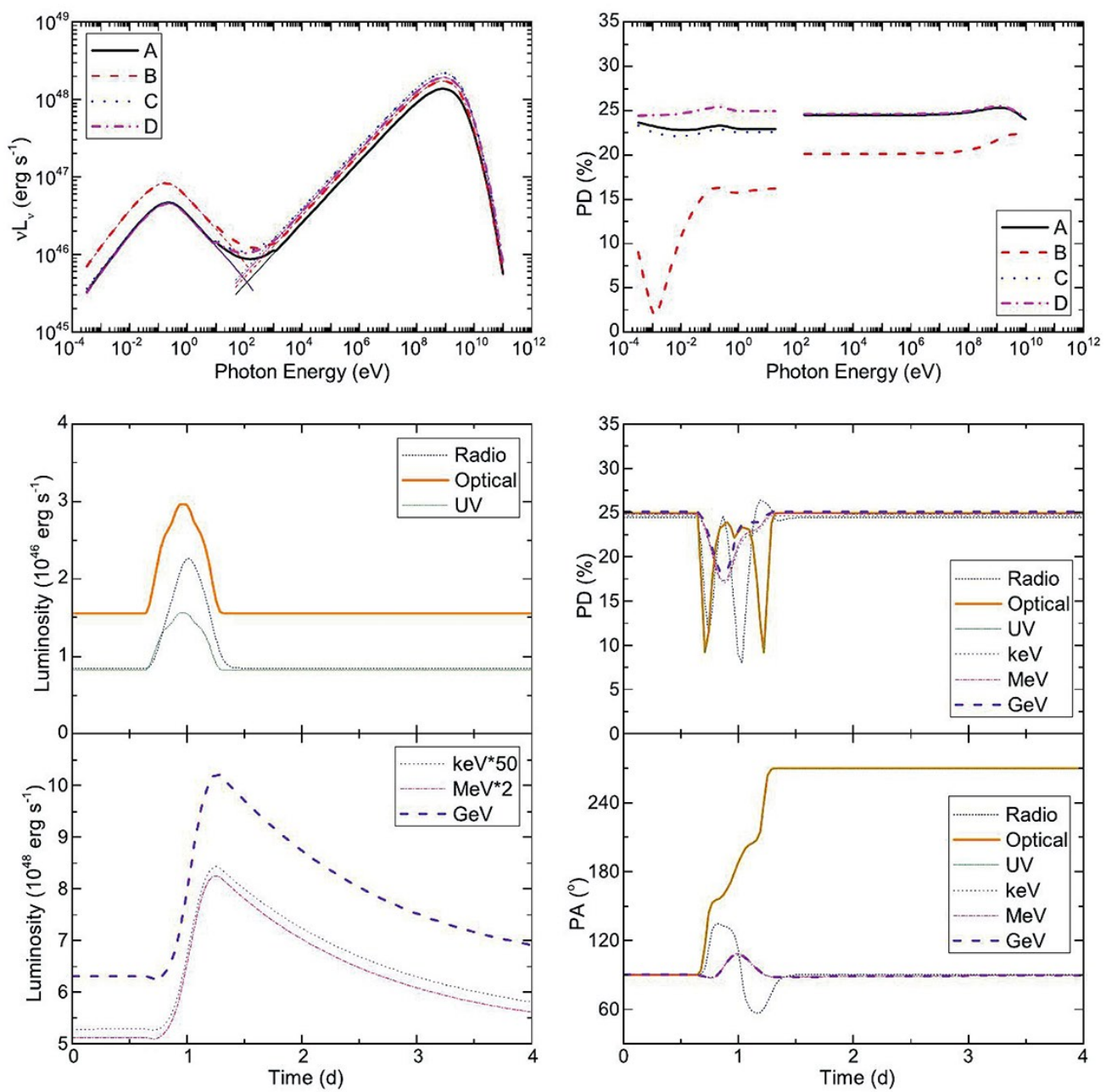

Figure 5. Radiation and polarization signatures from a proton-synchrotron-dominated lepto-hadronic shock-in-jet model, in a scenario where flaring activity is initiated by more efficient particle acceleration accompanied by a decrease of the magnetic field (magnetic energy dissipation), and where the light crossing time across the jet is shorter than the characteristic radiative cooling time of the highest-energy protons. Top left: Snap-shot SEDs for (A) the pre-flare equilibrium state, (B) a period shortly before the peak of the flare, (C) the peak of the flare, and (D) during the decay phase after the flare peak. Top right: Polarization Degree (PD) as a function of photon energy for the same epochs as the SEDs shown in the top-left panel. $C$ enter and bottom left: Multi-wavelength light curves; center and bottom right: Time-dependent Polarization Degree (PD) and Polarization Angle (PA) at various photon energies. From [33].

Acknowledgments: Markus Böttcher acknowledges support through the South African Research Chairs Initiative of the South African Department of Science and Technology and the National Research Foundation (Any opinion, finding and conclusion or recommendation expressed in this material is that of the authors, and the NRF does not accept any liability in this regard.) under SARChI grant No. 64789. This work has also been supported by NASA through Astrophysics Theory Program grant NNX10AC79G. 
Conflicts of Interest: The author declares no conflict of interest.

\section{References}

1. Marscher, A.M.; Gear, W.K. Models for high-frequency radio outbursts in extragalactic sources, with application to the early 1983 millimeter-to-infrared flare of 3C 273. Astrophys. J. 1985, 298, 114-127.

2. Böttcher, M.; Dermer, C.D. Timing signatures of the internal-shock model for blazars. Astrophys. J. 2010, 711, 445-460.

3. Chen, X.; Fossati, G.; Liang, E.P.; Böttcher, M. Time-dependent simulations of multiwavelength variability of the blazar Mrk 421 with a Monte Carlo multizone code. MNRAS 2011, 416, 2368-2387.

4. Chen, X.; Fossagi, G.; Böttcher, M.; Liang, E.P. Time-dependent simulations of emission from the FSRQ PKS 1510-089: Multiwavelength variability of external Compton and synchrotron self-Compton models. Mon. Not. R. Astron. Soc. 2012, 424, 789-799.

5. Graff, P.B.; Georganopoulos, M.; Perlman, E.S.; Kazanas, D. A multizone model for simulating the high-energy variability of TeV blazars. Astrophys. J. 2008, 689, 68-78.

6. Joshi, M.; Böttcher, M. Time-dependent radiation transfer in the internal shock model scneario for blazar jets. Astrophys. J. 2011, 727, 21.

7. Mimica, P.; Aloy, M.; Müller, E.; Brinkmann, W. Synthetic X-ray light curves of BL Lacs from relativistic hydrodynamic simulations. Astron. Astrophys. 2004, 418, 947-958.

8. Sokolov, A.; Marscher, A.P.; McHardy, I.M. Synchrotron self-Compton model for rapid nonthermal flares in blazars with frequency-dependent time lags. Astrophys. J. 2004, 613, 725-746.

9. Sokolov, A.; Marscher, A.P. External Compton radiation from rapid nonthermal flares in blazars. Astrophys. J. 2005, 629, 52-60.

10. Spada, M.; Ghisellini, G.; Lazzadi, D.; Celotti, A. Internal shocks in the jets of radio-loud quasars. Mon. Not. R. Astron. Soc. 2001, 325, 1559-1570.

11. Baring, M.G.; Böttcher, M.; Summerlin, E.J. Probing acceleration and turbulence at relativistic shocks in blazar jets. Mon. Not. R. Astron. Soc. 2016, submitted.

12. Summerlin, E.J.; Baring, M.G. Diffusive acceleration of particles at oblique, relativistic magnetohydrodynamic shocks. Astrophys. J. 2012, 745, 63.

13. Böttcher, M.; Reimer, A.; Sweeney, K.; Prakash, A. Leptonic and hadronic modeling of Fermi-detected blazars. Astrophys. J. 2013, 768, 54.

14. Abdo, A.A.; Ackermann, M.; Ajello, M.; Axelsson, M.; Baldini, L.; Ballet, J.; Barbiellini, G.; Bastieri, D.; Baughman, B.M.; Bechtol, K.; et al. A change in the optical polarization associated with a $\gamma$-ray flare in the blazar 3C 279. Nature 2010, 463, 919-923.

15. Marscher, A.P.; Jorstad, S.G.; D'Arcangelo, F.D.; Smith, P.S.; Williams, G.G.; Larionov, V.M.; Oh, H.; Olmstead, A.R.; Aller, M.F.; Aller, H.D.; et al. The inner jet of an active galactic nucleus revealed by a radio-to- $\gamma$-ray outburst. Nature 2008, 452, 966-969.

16. Marscher, A.P. Turbulent, extreme multi-zone model for simulating flux and polarization variability in blazars. Astrophys. J. 2014, 780, 87.

17. Zhang, H.; Chen, X.; Böttcher, M. Synchrotron polarization in blazars. Astrophys. J. 2014, 789, 66.

18. Larionov, V.M.; Jorstad, S.G.; Marscher, A.P.; Morozova, D.A.; Hagen-Thorn, V.A.; Konstantinova, T.S.; Kopatskaya, E.N.; Larionova, L.V.; Larionova, E.G.; Troitsky, I.S. The outburst of the blazar S5 0716+71 in 2011 October: Shock in a helical jet. Astrophys. J. 2013, 768, 40.

19. Ghisellini, G.; Tavecchio, L.; Ghirlanda, G.; Maraschi, L.; Celotti, A. General physical properties of bright Fermi blazars. Mon. Not. R. Astron. Soc. 2010, 402, 497-518.

20. Blinov, D.; Pavlidou, V.; Papadakis, I.E.; Hovatta, T.; Pearson, T.J.; Liodakis, I.; Panopoulou, G.V.; Angelakis, E.; Baloković, M.; Das, H.; et al. RoboPol: Optical polarization-plane rotations and flaring activity in blazars. Mon. Not. R. Astron. Soc. 2016, 457, 2252-2262.

21. Zhang, H.; Chen, X.; Boettcher, M.; Guo, F.; Li, H. Polarization swings reveal magnetic energy dissipation in blazars. Astrophys. J. 2015, 804, 58.

22. Abdo, A.A.; Ackermann, M.; Ajello, M.; Allafort, A.; Baldini, L.; Ballet, J.; Barbiellini, G.; Baring, M.G.; Bastieri, D.; Bechtol, K.; et al. Inights into the high-energy $\gamma$-ray emission of Markarian 501 from extensive multifrequency observations in the Fermi era. Astrophys. J. 2011, 727, 129. 
23. Ackermann, M.; Ajello, M.; Ballet, J.; Barbiellini, G.; Bastieri, D.; Bellazzini, R.; Blandford, R.D.; Bloom, E.D.; Bonamente, E.; Borgland, A.W.; et al. Multiwavelength observations of blazar AO 0235+164 in the 2008-2009 flaring state. Astrophys. J. 2012, 751, 159.

24. Sikora, M.; Madejski, G.; Moderski, R.; Poutanen, J. Learning about active galactic nucleus jets from spectral properties of blazars. Astrophys. J. 1997, 484, 108-117.

25. Cerruti, M.; Zech, A.; Boisson, C.; Inoue, S. A hadronic origin for ultra-high-frequency-peaked BL Lac objects. Mon. Not. R. Astron. Soc. 2015, 448, 910-927.

26. Mannheim, K.; Biermann, P.L. Gamma-ray flaring of 3C 279-A proton-initiated cascade in the jet? Astron. Astrophys. 1992, 253, L21-L24.

27. Mastichiadis, A.; Petropoulou, M.; Dimitrakoudis, S. Mrk 421 as a case study for TeV and X-ray variability in leptohadronic models. Mon. Not. R. Astron. Soc. 2013, 434, 2684-2695.

28. Mücke, A.; Protheroe, R.J. A proton synchrotron blazar model for flaring in Markarian 501. Astropart. Phys. 2001, 15, 121-136.

29. Mücke, A.; Protheroe, R.J.; Engel, R.; Rachen, J.P.; Stanev, T. BL Lac objects in the synchrotron proton blazar model. Astropart. Phys. 2003, 18, 593-613.

30. Petropoulou, M.; Mastichiadis, A. On proton synchrotron blazar models: The case of quasar 3C279. Mon. Not. R. Astron. Soc. 2012, 426, 462-472.

31. Petropoulou, M.; Dimitrakoudis, S.; Padovani, P.; Mastichiadis, A.; Resconi, E. Photohadronic origin of $\gamma$-ray BL Lac emission: Implications for IceCube neutrinos. Mon. Not. R. Astron. Soc. 2015, 448, 2412-2429.

32. Diltz, C.S.; Böttcher, M.; Fossati, G. Time dependent hadronic modeling of flat spectrum radio quasars. Astrophys. J. 2015, 802, 133.

33. Zhang, H.; Diltz, C.S.; Böttcher, M. Radiation and polarization signatures of a 3C multi-zone, time-dependent hadronic blazar model. Astrophys. J. 2016, submitted.

34. Zhang, H.; Böttcher, M. X-ray and gamma-ray polarization in leptonic and hadronic jet models of blazars. Astrophys. J. 2013, 774, 18.

(C) 2016 by the author; licensee MDPI, Basel, Switzerland. This article is an open access article distributed under the terms and conditions of the Creative Commons Attribution (CC-BY) license (http://creativecommons.org/licenses/by/4.0/). 